\title{
Severe hypertriglyceridemia in Norway: prevalence, clinical and genetic characteristics
}

\author{
Kjetil Retterstøl ${ }^{1,2}$, Ingunn Narverud ${ }^{1,3}$, Randi Selmer ${ }^{4}$, Knut E. Berge ${ }^{5}$, Ingvild V. Osnes ${ }^{1}$, Stine M. Ulven ${ }^{1}$,
} Bente Halvorsen ${ }^{6,7}$, Pål Aukrust ${ }^{6,7,8}$, Kirsten B. Holven ${ }^{1,3}$ and Per O. Iversen ${ }^{1,9^{*}}$

\begin{abstract}
Background: There is a lack of comprehensive patient-datasets regarding prevalence of severe hypertriglyceridemia (sHTG; triglycerides $\geq 10 \mathrm{mmol} / \mathrm{L}$ ), frequency of co-morbidities, gene mutations, and gene characterization in sHTG. Using large surveys combined with detailed analysis of sub-cohorts of sHTG patients, we here sought to address these issues.

Methods: We used data from several large Norwegian surveys that included 681,990 subjects, to estimate the prevalence. Sixty-five sHTG patients were investigated to obtain clinical profiles and candidate disease genes. We obtained peripheral blood mononuclear cells (PBMC) from six male patients and nine healthy controls and examined expression of mRNAs involved in lipid metabolism.

Results: The prevalence of sHTG was 0.13 (95\% Cl 0.12-0.14)\%, and highest in men aged 40-49 years and in women 60-69 years. Among the 65 sHTG patients, a possible genetic cause was found in four and 11 had experienced acute pancreatitis. The mRNA expression levels of carnitine palmitoyltransferase (CPT)-1A, CPT2, and hormone-sensitive lipase, were significantly higher in patients compared to controls, whereas those of ATP-binding cassette, sub-family G, member 1 were significantly lower.

Conclusions: In Norway, sHTG is present in $0.1 \%$, carries considerable co-morbidity and is associated with an imbalance of genes involved in lipid metabolism, all potentially contributing to increased cardiovascular morbidity in sHTG.
\end{abstract}

Keywords: Co-morbidity, Genetics, Pancreatitis, Prevalence, Triglycerides

\section{Background}

Severe hypertriglyceridemia (sHTG) can be defined as a non-fasting serum concentration of triglycerides (TG) $\geq 10 \mathrm{mmol} / \mathrm{L}$. The frequency of risk factors for $\mathrm{sHTG}$ (e.g. obesity, diabetes and high alcohol consumption) has increased in the last decades, calling for increased awareness of sHTG, and at present the prevalence in the general population is still uncertain.

There are upcoming possible therapies for sHTG such as apolipoprotein CIII inhibitors or lopitapide [1]. In addition, a gene therapy product for lipoprotein lipase deficiency has been approved by the European Medicines Agency [2], and monoclonal antibody-based therapy has also been applied [3]. To utilize these novel therapeutic modalities there is a need for more data on the prevalence and consequences of sHTG.

In the present study we therefore aimed (i) to describe the prevalence of sHTG in Norway by surveying several large cohorts; (ii) to present detailed clinical data of patients with sHTG; and (iii) to examine the expression of lipid-related genes in peripheral blood mononuclear cells of patients with hypertriglyceridemia.

\footnotetext{
* Correspondence: p.o.iversen@medisin.uio.no

${ }^{1}$ Department of Nutrition, Institute of Basic Medical Sciences, University of Oslo, P.O. Box 1046Blindern, 0317 Oslo, Norway

${ }^{9}$ Department of Haematology, Oslo University Hospital, Oslo, Norway

Full list of author information is available at the end of the article
}

\section{Methods}

The study protocol was approved by the Regional Committee for Medical Health Research Ethics (\#S-08337a/ S-07455a) and by the Norwegian Data Inspectorate 
(\#08/3914). A written informed consent was obtained from each patient included in the study and the study protocol conformed to the ethical guidelines of the 1975 Declaration of Helsinki.

\section{Collection of data from three surveys to estimate the prevalence of sHTG}

The current data on prevalence originates from (i) the COhorts of NORway (CONOR; $n=172,956)$; (ii) the 3 Counties Study ( $n=92,080)$; and (iii) the 40 Years Survey $(n=416,954)$. The data from these three surveys have in the present study been merged $(n=681,990)$ to generate data of the Norwegian population.

CONOR covers 10 population-based studies during 1994-2003 from different parts of Norway, both urban and rural regions. Some studies invited all residents above a specific age (for example all above 19 years in the HUNT 2 study), whereas others invited all subjects in selected age groups (for example all 30-, 40-, 45-, 60 and 75 years in the OPPHED- and TROFINN studies). The overall participation rate was $60 \%$ and varied between the studies. The participants (aged 20-103 years) filled in a questionnaire covering physical activity, history of previous cardiovascular disease (CVD), diabetes and use of medicine. A non-fasting blood sample was collected. Body mass index (BMI) and systolic blood pressure were also measured. For subjects who participated more than once we used the last measured TG values. Details of the research tools and the measurements of blood chemistry have been described elsewhere [4].

In the 3 Counties Study (covering the counties Oppland in Eastern part of Norway; Sogn og Fjordane in Western part of Norway; and Finnmark in Northern part of Norway; during 1974-1988) all residents aged 3549 years at the study start in each county were invited to the first screening. The study had three visits in each county. The same birth cohorts and in some counties broader age groups, were invited to the second and third screening in each county. Altogether about 93,000 men and women participated in at least one screening. The participation rate varied from $78 \%$ to $90 \%$. The participants were screened for CVD risk factors and a nonfasting blood sample was collected. We have included the last measured TG value for each participant in the present study. Details of the research tools and the measurements of blood chemistry have been described elsewhere $[5,6]$.

The 40 Years Survey was conducted from 1985 to 1999 and consisted of 429,245 participants from 18 counties. All inhabitants aged 40-42 years were invited to the survey whereas in some counties and municipalities broader age groups were included. The participation rate varied from 69\% in Nord-Trøndelag county in 1992 to $46 \%$ in Østfold- and Aust-Agder counties in 1999. A non-fasting blood sample was collected. A few subjects participated more than once, and for them the last TG value was used in the present study. Details of the research tools and the measurements of blood chemistry have been described elsewhere [7].

From each study, we included all participants who had obtained measurements of serum triglycerides and who had answered the questionnaires.

\section{Collection of data from patients with sHTG for detailed analyses}

All individuals $>18$ years treated for sHTG at the Lipid Clinic from January 1st 2002 to December 31st 2007 were identified by searching the medical records for the diagnoses (ICD-10 classification): pure hypertriglyceridemia (E78.1), mixed hyperlipidemia (E78.2) and hyperchylomicronemia (E78.3). To ensure that the hypertriglyceridemia was not transient, only patients with long-lasting sHTG was included, i.e. they were diagnosed with sHTG both at the time of referral and at two consecutive time points 2-6 months apart. One hundred and twelve patients fulfilled the inclusion criteria and were invited by letter. Sixty-five (58\%) patients returned a signed informed consent and were included in the study and came for a baseline visit. Inclusion and exclusion criteria are listed in the Additional file 1: Table S1. Data regarding age, diagnoses, clinical findings, laboratory measures, and use of medication were collected from their medical records. All laboratory values were collected after at least $12 \mathrm{~h}$ of fasting and analysed at the laboratory at Oslo University Hospital, using standard procedures. Most patients were controlled after $0.5,1,2$ and 3 years $( \pm$ 3 months) after the baseline visit. The genes lipoprotein lipase (LPL), apolipoprotein C-II (APOC2), apolipoprotein A-V (APOA5), glycosylphosphatidylinositol-anchored high density lipoprotein-binding protein 1 (GPIHBP1) and lipase maturation factor 1 (LMF1) were sequenced at the Unit for Cardiac and Cardiovascular Genetics, Department of Medical Genetics, Oslo University Hospital, and apolipoprotein E was genotyped. Information regarding diet and lifestyle were collected from the validated food questionnaire SmartDiet [8].

\section{RNA expression of lipid-related genes in peripheral blood mononuclear cells}

Six male patients with hypertriglyceridemia and nine male control subjects were recruited from employees of the Lipid Clinic and the University of Oslo, respectively, to this sub-study.

We analyzed a range of lipid-related genes (Additional file 1: Table S2) in peripheral blood mononuclear cells (PBMC) obtained from heparinized blood by gradient centrifugation in Isopaque-Ficoll (Lymphoprep, Nycomed, Oslo, Norway). The assay-numbers (hs-numbers) of the 
specific genes chosen for the analysis are included (Additional file 1: Table S2). PBMC pellets for mRNA expression analyses were immediately frozen and stored at $-80{ }^{\circ} \mathrm{C}$. Total RNA was isolated from all PBMC samples using RNeasy mini kit (Qiagen, Hilden, Germany) with lysis buffer containing $\beta$-mercaptoethanol, and RNase-Free DNase (Qiagen) and stored at $-80{ }^{\circ} \mathrm{C}$. RNA quantity and -quality measurements were performed using the ND 1000 Spectrophotometer (Saveen Werner, Carlson Circle Tampa, FL) and Agilent Bioanalyser (Agilent Technologies, Santa Clara, CA), respectively. Four hundred ng RNA from all samples were reversetranscribed using High Capacity RNA-to-cDNA Kit (Applied Biosystems, Foster City, CA). Quantitative real-time polymerase chain reaction (RT-qPCR) was performed using Custom TaqMan Array micro Fluidic cards (Applied Biosystems). The endogenous control genes glucuronidase $\beta$ (GUS $\beta$ ) and TATA box binding protein $(T B P)$ were used for normalization. The relative mRNA level for each transcript was calculated by the $\Delta \Delta$ cycle threshold method [9].

\section{Statistical analyses}

The patient data were analysed with the Statistical Package for the Social Sciences (SPSS), version 16.0. Results from non-parametric statistical analyses are reported as median and 95\% confidence interval (95\% CI). Results from variables that showed a normal distribution are reported as mean and standard deviation (SD). For comparisons of two groups of individuals, the MannWhitney $U$ test was used. Significance was assumed for $p<0.05$. The prevalence data were analyzed with SPSS version 19.

\section{Results}

\section{Prevalence of hypertriglyceridemia}

The prevalence of subjects with non-fasting serum TG concentrations $\geq 10 \mathrm{mmol} / \mathrm{L}$, i.e. sHTG, was 0.13 (95\% CI $0.12-0.14) \%$ based on pooled data from the three different surveys, while $2.0 \%$ of the participants had moderately increased TG levels (i.e. between 5.0 and $9.9 \mathrm{mmol} / \mathrm{L}$ ) (Table 1). Furthermore, about $28 \%$ had TG levels above $2 \mathrm{mmol} / \mathrm{L}$, a cut-off value often used for diagnosing hypertriglyceridemia [10]. Notably these pooled estimates stem from different surveys with somewhat different inclusion criteria, age distributions and time periods.

\section{Hypertriglyceridemia in the CONOR survey- relation to other risk factors}

The CONOR survey provided further age- and gender specific data of the included participants. The prevalence of sHTG was significantly higher in men than in women for the three age groups between 30 and 59 years (Table 2 ). Moreover, the prevalence peaked in the age group 40-49 years for men and in the age group 60-69 years for women. We next examined whether various known risk factors for cardiovascular disease were associated with elevated TG values (Table 3). A non-fasting serum concentration of glucose $\geq 11 \mathrm{mmol} / \mathrm{L}$ was present in $15.4 \%$ of those with sHTG, compared to only $0.7 \%$ of subjects with non-fasting TG $<2 \mathrm{mmol} / \mathrm{L}(p<0.05)$ indicating that high serum glucose was more than 20 times more prevalent in sHTG subjects. The prevalence of systolic blood pressure above $160 \mathrm{mmHg}$ was similar in those with sHTG (13.9\%) and those with non-fasting TG $<2 \mathrm{mmol} / \mathrm{L}(10.2 \%)(p>0.05)$. Furthermore, among the sHTG subjects, a BMI $\geq 30 \mathrm{~kg} / \mathrm{m}^{2}$ was present in $41.6 \%$ in comparison to $10.8 \%$ of subjects with nonfasting TG $<2 \mathrm{mmol} / \mathrm{L}(p<0.05)$. When asked if they had ever experienced a myocardial infarction (MI), $2.7 \%$ of the subjects with TG $<2 \mathrm{mmol} / \mathrm{L}$ answered yes compared to $5.8 \%$ of those with sHTG $(p<0.05)$. Only $6 \%$ of sHTG subjects reported to be physical active $\geq 3 \mathrm{~h} /$ week compared to $13.7 \%$ among those with TG $<2 \mathrm{mmol} / \mathrm{L}$ $(p<0.05)$. Daily smoking was reported by $29.9 \%$ of subjects with TG levels $<2 \mathrm{mmol} / \mathrm{L}$ and in $48.6 \%$ of those with sHTG $(p<0.05)$.

\section{Clinical, genetic and biochemical profiles of patients with severe hypertriglyceridemia}

To give a more in-depth characterization of patients with sHTG, 65 subjects who agreed to participate (51 men and 14 women) were further characterized by clinical, genetic and biochemical methods. Among these 65

Table 1 Distribution of non-fasting serum triglycerides in Norwegian population surveys

\begin{tabular}{|c|c|c|c|c|c|}
\hline \multirow{3}{*}{ Survey } & \multirow[b]{2}{*}{ Participants ${ }^{a}$} & \multicolumn{4}{|c|}{ Triglyceride concentration $(\mathrm{mmol} / \mathrm{L})$} \\
\hline & & $<2.0$ & $2.0-4.9$ & $5.0-9.9$ & $\geq 10.0$ \\
\hline & $n$ & $n(\%)$ & $n(\%)$ & $n(\%)$ & $n(\%)$ \\
\hline CONOR & 172,956 & $125,455(72.54)$ & $44,408(25.68)$ & $2919(1.69)$ & $174(0.10)$ \\
\hline 3 Counties Study & 92,080 & $62,928(68.34)$ & $27,071(29.40)$ & $1955(2.12)$ & $126(0.14)$ \\
\hline 40 Years Survey & 416,954 & $299,080(71.73)$ & $108,615(26.05)$ & $8695(2.09)$ & $562(0.13)$ \\
\hline Total & 681,990 & $487,463(71.48)$ & $180,094(26.41)$ & $13,569(1.99)$ & $862(0.13)$ \\
\hline
\end{tabular}

${ }^{\mathrm{a}}$ For persons who participated more than once, the last triglyceride value was used in the present study 
Table 2 Prevalence of severe hypertriglyceridemia in the CONOR survey

\begin{tabular}{|c|c|c|c|c|c|c|}
\hline \multirow[b]{2}{*}{ Age group (years) } & \multicolumn{3}{|l|}{ Men } & \multicolumn{3}{|c|}{ Women } \\
\hline & $\overline{\text { SHTG }}$ & $n$ & Prevalence $^{\mathrm{a}}(95 \% \mathrm{Cl})$ & $\overline{\text { SHTG }}$ & $n$ & Prevalence $^{\text {a }}(95 \%$ Cl) \\
\hline $0-29$ & 2 & 5274 & $38(5-137)$ & 1 & 6458 & $15(0-86)$ \\
\hline $30-39$ & 22 & 12,921 & $170(107-258)$ & 1 & 15,126 & $7(0-37)$ \\
\hline $40-49$ & 73 & 28,156 & $259(203-326)$ & 16 & 32,376 & $49(28-80)$ \\
\hline $50-59$ & 21 & 9847 & $213(132-326)$ & 3 & 9687 & $31(6-90)$ \\
\hline $60-69$ & 12 & 12,278 & $98(51-171)$ & 10 & 10,890 & $92(44-169)$ \\
\hline $70-79$ & 5 & 13,925 & $36(12-84)$ & 6 & 11,879 & $51(19-110)$ \\
\hline $80-89$ & 0 & 1604 & ND & 2 & 2535 & $79(10-285)$ \\
\hline
\end{tabular}

The prevalence data are expressed per 100,000 subjects. sHTG severe hypertriglyceridemia, $\mathrm{Cl}$ confidence interval, $N D$ not detected

Table 3 Distribution of biochemistry, clinical and lifestyle characteristics in relation to serum triglycerides in the CONOR survey

\begin{tabular}{|c|c|c|c|c|c|c|c|c|c|c|}
\hline & \multicolumn{10}{|c|}{ Non-fasting trigycerides (mmol/L) } \\
\hline & \multicolumn{2}{|l|}{$<2.0$} & \multicolumn{2}{|l|}{$2.0-4.9$} & \multicolumn{2}{|l|}{$5.0-9.9$} & \multicolumn{2}{|c|}{$\geq 10$} & \multicolumn{2}{|l|}{ Total } \\
\hline & $n$ & $\%$ & $n$ & $\%$ & & $\%$ & $n$ & $\%$ & & $\%$ \\
\hline \multicolumn{11}{|c|}{ Non-fasting serum glucose (mmol/L) } \\
\hline$<7.0$ & 103,889 & 94.5 & 35,527 & 88.0 & 2134 & 80.2 & 119 & 73.5 & 141,669 & 92.5 \\
\hline $7.0-8.9$ & 4432 & 4.0 & 3065 & 7.6 & 262 & 9.8 & 16 & 9.9 & 7775 & 5.1 \\
\hline $9.0-10.9$ & 855 & 0.8 & 759 & 1.9 & 95 & 3.6 & 2 & 1.2 & 1711 & 1.1 \\
\hline$\geq 11.0$ & 734 & 0.7 & 1006 & 2.5 & 170 & 6.4 & 25 & 15.4 & 1935 & 1.3 \\
\hline Total & 109,910 & 100.0 & 40,357 & 100.0 & 2661 & 100.0 & 162 & 100.0 & 153,090 & 100.0 \\
\hline \multicolumn{11}{|c|}{ Systolic blood pressure (mmHg) } \\
\hline$<160$ & 112,395 & 89.8 & 36,850 & 83.3 & 2376 & 81.5 & 149 & 86.1 & 151,770 & 88.0 \\
\hline$\geq 160$ & 12,715 & 10.2 & 7383 & 16.7 & 538 & 18.5 & 24 & 13.9 & 20,660 & 12.0 \\
\hline Total & 125,110 & 100.0 & 44,233 & 100.0 & 2914 & 100.0 & 173 & 100.0 & 172,430 & 100.0 \\
\hline \multicolumn{11}{|c|}{ Body mass index $\left(\mathrm{kg} / \mathrm{m}^{2}\right)$} \\
\hline$<20$ & 6120 & 4.9 & 350 & 0.8 & 5 & 0.2 & 0 & 0.0 & 6475 & 3.8 \\
\hline $20.0-24.9$ & 58,443 & 46.8 & 9791 & 22.2 & 339 & 11.7 & 16 & 9.2 & 68,589 & 39.9 \\
\hline $25.0-29.9$ & 46,690 & 37.4 & 22,380 & 50.8 & 1464 & 50.5 & 85 & 49.1 & 70,619 & 41.1 \\
\hline$\geq 30.0$ & 13,501 & 10.8 & 11,506 & 26.1 & 1092 & 37.7 & 72 & 41.6 & 26,171 & 15.2 \\
\hline Total & 124,754 & 100.0 & 44,027 & 100.0 & 2900 & 100.0 & 173 & 100.0 & 171,854 & 100.0 \\
\hline \multicolumn{11}{|c|}{ Have had myocardial infarction } \\
\hline Yes & 3371 & 2.7 & 2193 & 5.0 & 146 & 5.1 & 10 & 5.8 & 5720 & 3.4 \\
\hline No & 120,420 & 97.3 & 41,489 & 95.0 & 2718 & 94.9 & 163 & 94.2 & 164,790 & 96.6 \\
\hline Total & 123,791 & 100.0 & 43,682 & 100.0 & 2864 & 100.0 & 173 & 100.0 & 170,510 & 100.0 \\
\hline \multicolumn{11}{|c|}{ Hard physical activity during one week } \\
\hline No & 38,733 & 36.5 & 15,432 & 43.0 & 1089 & 46.0 & 58 & 38.4 & 55,312 & 38.3 \\
\hline$<1 \mathrm{~h}$ & 26,506 & 25.0 & 9176 & 25.6 & 597 & 25.2 & 50 & 33.1 & 36,329 & 25.1 \\
\hline $1-2 h$ & 26,371 & 24.8 & 7328 & 20.4 & 449 & 19.0 & 34 & 22.5 & 34,182 & 23.6 \\
\hline$\geq 3 \mathrm{~h}$ & 14,575 & 13.7 & 3917 & 10.9 & 233 & 9.8 & 9 & 6.0 & 18,734 & 13.0 \\
\hline Total & 106,185 & 100.0 & 35,853 & 100.0 & 2368 & 100.0 & 151 & 100.0 & 144,557 & 100.0 \\
\hline \multicolumn{11}{|c|}{ Daily smoking } \\
\hline No & 87,015 & 70.1 & 30,394 & 69.4 & 1862 & 64.7 & 89 & 51.4 & 119,360 & 69.8 \\
\hline Yes & 37,129 & 29.9 & 13,380 & 30.6 & 1016 & 35.3 & 84 & 48.6 & 51,609 & 30.2 \\
\hline Total & 124,144 & 100.0 & 43,774 & 100.0 & 2878 & 100.0 & 173 & 100.0 & 170,969 & 100.0 \\
\hline
\end{tabular}


patients, 28 were diagnosed with a primary hypertriglyceridemia whereas the remaining 37 had a secondary hypertriglyceridemia.

Among the men, the mean ( \pm SD) age was $45.6 \pm 9.1$ years and the mean BMI was $29.2 \pm 4.0 \mathrm{~kg} / \mathrm{m}^{2}$. Among the women, the mean age was $46.7 \pm 6.4$ years and the mean BMI was $29.4 \pm 4.6 \mathrm{~kg} / \mathrm{m}^{2}$. In four of the 28 patients with primary hypertriglyceridemia a possible genetic cause of the sHTG was found: Homozygosity for a deletion comprising exons 3 and 4 in GPIHBP1 was found in two women who both had been hospitalized for pancreatitis several times [11]. Another patient suffered from type III hyperlipidemia due to apolipoprotein E2/E2 homozygosity. One patient was heterozygous for the two non-synonymous SNPs: c.106G > A (rs1801177) and c.998G > A (rs144466625) (Ref. seq.: NM _000237.2) in the $L P L$ gene. We do not know whether these SNPs reside on different alleles. No mutations were found in $L M F 1$, APOC2 or APOA5.

The values of the biochemical characteristics of the 65 patients with sHTG were within the standard reference limits except for high values of total cholesterol and TG (Additional file 1: Table S3). The median and maximum fasting serum TG values at the first visit were 12.3 and $57.3 \mathrm{mmol} / \mathrm{L}$, respectively. Forty-eight percent of the patients smoked daily, $50 \%$ were overweight $\left(\mathrm{BMI}>25 \mathrm{~kg} / \mathrm{m}^{2}\right), 36 \%$ were obese $\left(\mathrm{BMI}>30 \mathrm{~kg} / \mathrm{m}^{2}\right)$, and $43 \%$ were physically inactive $(<30 \mathrm{~min}$ of physical activity maximally once per week).

Metabolic syndrome, hypertension, high alcohol intake, diabetes mellitus type 2 and other conditions known to be associated with sHTG were observed in 61 of the 65 (94\%) patients (Fig. 1). In total, 11 (17\%) patients had experienced pancreatitis (4 with one episode and 7 with recurrent episodes) and all had co-factors known to increase the risk for pancreatitis (alcohol abuse, $n=4$ and diabetes type $2, n=7$ ). At the first measurement after initiation of treatment, patients who

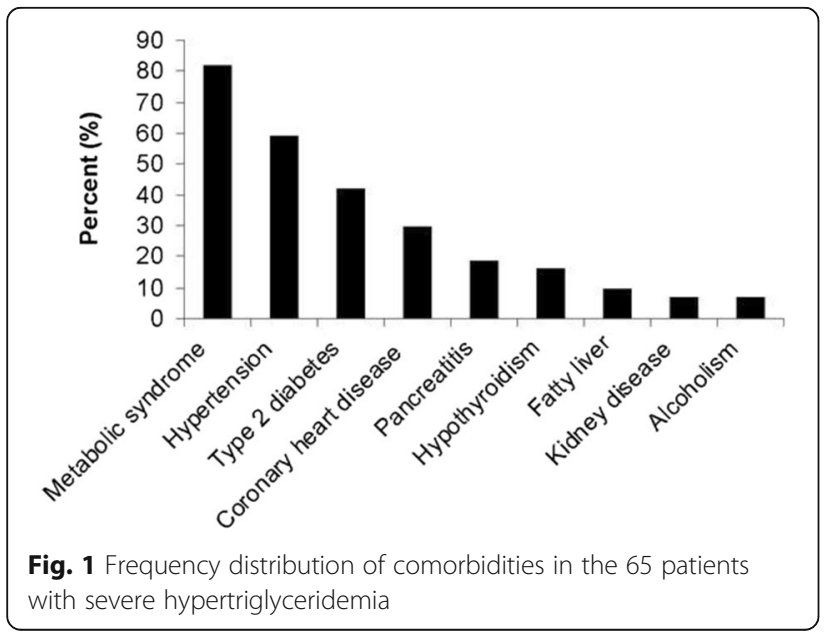

had experienced acute pancreatitis (mean TG $22.1 \mathrm{mmol} / \mathrm{L}$ ) had significantly higher TG values than patients without pancreatitis (mean TG $8.2 \mathrm{mmol} / \mathrm{L}$ ). Eleven of the patients with sHTG had a medical history of coronary artery disease and eight other patients experienced a coronary event during the study period.

All 65 patients had received lifestyle advice and diet therapy from a clinical nutritionist at least once, whereas 33 used one or several lipid-lowering medications when referred to the Lipid Clinic, increasing to 43 at the last consultation (Additional file 1: Table S4). None used medication known to increase TG. Among the 65 patients, 10 had reached a serum TG concentration within the laboratory reference value of $2.6 \mathrm{mmol} / \mathrm{L}$ or lower one year after the first visit at the Lipid Clinic. Due to the small number of this subset compared to the much higher number in the larger surveys, we cannot readily make comparisons between them.

\section{PBMC expression of lipid-related genes}

In the subgroup of six male hypertriglyceridemic patients and 9 male control subjects, mRNA levels of relevant genes involved in lipid metabolism were analysed in PBMC since previous studies have shown that dyslipidemic alterations are reflected in the PBMC transcriptome $[12,13]$. Their characteristics are displayed in the Additional file 1: Table S5. None of them had cardiovascular disease. These patients had significantly higher levels of total cholesterol, TG and glucose, and lower levels of apolipoprotein A1 compared to the controls (Table 4).

The mRNA expression levels of $C P T-1 A$ and $C P T-2$, encoding mitochondrial enzymes involved in $\beta$-oxidation of fatty acids, were significantly higher in the patients compared to the controls (Additional file 2: Figure S1). The mRNA expression of LIPE, encoding the hormone sensitive lipase that hydrolyzes stored TG to free fatty acids, was also significantly elevated among the patients compared to the controls. In contrast, the mRNA expression of $A B C G 1$, encoding a protein contributing to cholesterol efflux capacity, was significantly lower in the HTG patients compared to the controls.

\section{Discussion}

In the present study we show that the prevalence of sHTG in Norway is about $0.1 \%$, and associated with a number of serious co-morbidities such as CVD and pancreatitis. The high prevalence of elevated non-fasting glucose concentrations, obesity and low physical activity suggest a disturbed energy metabolism in these subjects. Moreover, in a subgroup of patients, we found that the hypertriglyceridemic phenotype was associated with changes in gene expression of key players in lipid metabolism, potentially contributing to the high prevalence of CVD in these patients. 
Table 4 Characteristics of the male sub-sample with gene expression profiling and cholesterol efflux measurements compared with male control subjects

\begin{tabular}{llll}
\hline & Hypertriglyceridemia & Controls & $p$-values \\
& $n=6$ & $n=9$ & 0.41 \\
\hline Age (years) & $44(32-50)$ & $52(25-60)$ & $0.5(3.7-5.6)$ \\
Total cholesterol (mmol/L) & $8.9(5.2-14.6)$ & $2.9(2.0-4.1)$ & 0.004 \\
LDL cholesterol (mmol/L) & $2.3(1.9-3.8)$ & $1.5(1.1-2.0)$ & 0.41 \\
HDL cholesterol (mmol/L) & $1.4(0.8-2.4)$ & $0.7(0.4-1.5)$ & 0.63 \\
Triglycerides (mmol/L) & $17.3(5.1-58)$ & $1.5(1.1-1.7)$ & 0.001 \\
Apolipoprotein A1 (mg/L) & $1.1(1.1-1.4)$ & $0.8(0.6-1.1)$ & 0.019 \\
Apolipoprotein B (mg/L) & $0.8(0.7-1.1)$ & $387(172-681)$ & 0.58 \\
Free fatty acids (nmol/L) & $593(372-798)$ & $1.1(0.7-2.8)$ & 0.090 \\
Hs-CRP (mg/L) & $2.4(0.7-20)$ & $5.0(4.4-5.4)$ & 0.20 \\
Glucose $(\mathrm{mmol} / \mathrm{L})$ & $10.1(5.5-14.7)$ & 0.001 &
\end{tabular}

Data are presented as median (range)

$H s$-CRP high sensitivity C-reactive protein

There are few nation-wide data on the prevalence of hypertriglyceridemia, and there is no established definition of sHTG. Reportedly $1.7 \%$ of adults in the United States 2001-2006 had elevated fasting TG values ranging from 5.6 to $22.6 \mathrm{mmol} / \mathrm{L}$ [14], whereas we found that $2 \%$ of the subjects had moderately elevated non-fasting TG levels $(5-10 \mathrm{mmol} / \mathrm{L})$ in Norway, similar to the $1.9 \%$ recently reported in Denmark [10]. In addition, about 25\% of US adults had hypertriglyceridemia (TG values $>2 \mathrm{mmol} / \mathrm{L}$ ), i.e. about the same estimate as we report here $(28 \%)$. In comparison, among otherwise healthy adult Iranians, nearly $10 \%$ had hypertriglyceridemia in 2013 [15], whereas the corresponding percentage of hypertriglyceridemia in Västerbotten County, Sweden in 2008-2010 was 15.5 and 31.9 among women and men, respectively [16]. In the present study $20 \%$ of the women and $36 \%$ of the men had TG values $>2 \mathrm{mmol} / \mathrm{L}$, based on the data from the CONOR survey. Collectively, our Norwegian data are quite similar to the quoted estimates of the prevalence of hypertriglyceridemia obtained in populations from the US [14], Iran [15] and Sweden [16]. Notably, these populations differ in relation to lifestyles and risks of CVD. Moreover, the data from the CONOR-survey consistently showed that sHTG level was associated with a number of risk factors for CVD, diabetes and the metabolic syndrome, as evidenced by elevated glucose, blood pressure, BMI as well as low physical activity [17]. If these risk factors represent potential causes or consequences of sHTG or if they are associated phenomena reflecting common pathogenic mechanisms such as those leading to metabolic syndromes, are at present not known.

The 28 patients with primary hypertriglyceridemia who were included in the sHTG sub-study had persistent sHTG for several months, but in only four of them was a possible genetic cause found, demonstrating that even in a long lasting sHTG a genetic cause is usually not found among currently known predisposing genes. Unfortunately we do not have data regarding the prevalence of heterozygous mutations in the 65 patients. The fasting TG values were reduced by nearly $60 \%$ after treatment, a reduction that seemed to persist for years. In line with others we observed that SHTG was associated with several serious co-morbidities [18]. Notably, 19 of the 65 patients (29\%) had established coronary heart disease and eight of these patients experienced a coronary event during the study period, suggesting that they were at significant risk even after treatment with marked decrease in TG levels. Without a control group we cannot exclude the possibility that the fall in TG levels is the natural evolution of the disease (sHTG). However, the relatively rapid effect of treatment observed after 6 months, which then remained fairly constant, may be interpreted as a real treatment effect. Similar to other studies [19,20] our data shows that complete normalization of plasma TG values is rarely achieved in patients with sHTG. In patients with sHTG, those with a history of acute pancreatitis had particularly high TG values. Moreover, patients with sTG levels and pancreatitis all had co-factors known to increase the risk for pancreatitis [18], potentially suggesting that sHTG on its own will not predispose to acute pancraetitis. On the other hand, Pedersen et al. [10] recently showed that even those with mild hypertriglyseridemia (TG $>2 \mathrm{mmol} /$ L) were at increased risk for acute pancreatitis, after adjustment for several known confounders.

In the subgroup of six hypertriglyceridemic patients we found that the PBMC mRNA expression of several key mediators in lipid metabolism was altered as compared to controls. Thus, the patients were characterized 
by higher mRNA expression of CPT-1A, CPT-2, and $L I P E$, concomitantly with lower mRNA expression of ABCG1 compared to controls. Defects in CPT-1 and CPT-2 are associated with hypertriglyceridemia in humans [21], whereas increased CPT-1 activity is associated with reduced TG concentrations and reduced muscle- and liver steatosis in rats [22], suggesting that the high TG levels in the present study population is not caused by decreased $\beta$-oxidation. CPT is a PPAR $\alpha$ target gene and, as expected, in the patients treated with fibrates, the PBMC mRNA expression of CPT1A and $C P T 2$ were higher compared to controls, thereby counteracting the increased TG levels. Hormone sensitive lipase (LIPE) is also a PPAR $\alpha$ target gene, inducing hydrolyses of TG to fatty acids, and this enzyme was also upregulated in PBMC from patients. Thus, it is tempting to hypothesize that the up-regulation of these PPAR $\alpha$ sensitive enzymes may represent counteracting mechanisms to down-regulate TG levels. In contrast to the upregulation of genes involved in fatty acid metabolism, PBMC from these patients had decreased mRNA expression of $A B C G 1$, a transporter involved in mediating cholesterol efflux, potentially leading to reduced cholesterol efflux capacity.

In the present study we combined data of prevalence and treatment with analyses to characterize the molecular etiology to strengthen the observations. We acknowledge the fact that since our pooled estimate of sHTG was based on several large surveys, we cannot conclude that this is fully representative for the general Norwegian population. Another possible weakness in large population surveys is that sick people usually participate to a lower extent than healthy. Additionally we cannot exclude possible confounding due to differences in life-styles. Since sHTG is associated with a considerable comorbidity the present data may thus have underestimated the true prevalence of sHTG in the population. On the other hand, patients with sHTG are referred to the Lipid Clinic from all over Norway increasing the representativeness for this population. A fasting TG value was not always available. Moreover, lack of a control group and a participation rate of $58 \%$ may have led to a selection bias in the 65 patients with persistent sHTG. Regarding the PBMC sub-study, a limitation is that few patients were included and only male subjects were studied.

\section{Conclusions}

In conclusion, sHTG was present in 1/1000 subjects based on a pooled estimate from several large surveys. Furthermore sHTG was associated with serious comorbidity, in particular CVD, and was associated with an imbalance of genes involved in lipid metabolism. Notably, sHTG is easy and inexpensive to detect and deserve increased attention to limit serious organ complications.

\section{Additional file}

Additional file 1: Table S1. Inclusion and exclusion criteria for patients with SHTG for detailed analyses. Table S2. List of lipid-related genes analyzed in peripheral blood mononuclear cells from patients with severe hypertriglyceridemia. Table S3. Baseline biochemical characteristic of 65 patients with severe hypertriglyceridemia. Table S4. Combinations of medications used at end of study among the 65 patients with hypertriglyceridemia. Table S5. Characteristics of the six patients with hypertriglyceridemia subjected to in-depth molecular analyses. (PDF $258 \mathrm{~kb}$ )

\section{Abbreviations}

ABCG1: ATP-binding cassette, sub-family G, member 1; APO: Apolipoprotein; BMI: Body mass index; CONOR: Cohorts of Norway; CPT: Carnitine palmitoyltransferase; CVD: Cardiovascular disease;

GPIHBP1: Glycosylphosphatidylinositol-anchored high density lipoproteinbinding protein 1; GUS $\beta$ : Glucuronidase $\beta$; LIPE: Hormone sensitive lipase; LMF1: Lipase maturation factor 1; LPL: Lipoprotein lipase; PBMC: Peripheral blood mononuclear cells; SD: Standard deviation; sHTG: Severe hypertriglyceridemia; TBP: TATA box binding protein; TG: Triglycerides

\section{Funding}

The study was supported in part by the Throne Holst Foundation for Nutrition Research, University of Oslo.

\section{Availability of data and materials}

All datasets generated and analyzed in this study are available from the corresponding author on request.

\section{Authors' contributions}

$\mathrm{KR}$ and POI planned the study and drafted the manuscript. IN, SMU, BH, PA and $\mathrm{KBH}$ performed the mRNA expression analyses. IVO collected data from the 65-patient sub-study. RS collected and analysed the data from the population surveys. KEB performed the genetic profiling. All authors analysed data and revised the manuscript. All authors read and approved the final manuscript.

\section{Competing interests}

The authors declare no conflict of interest with respect to this manuscript.

Consent for publication

Not applicable.

\section{Ethics approval and consent to participate}

The study protocol was approved by the Regional Committee for Medical Health Research Ethics (\#S-08337a/S-07455a) and by the Norwegian Data Inspectorate (\#08/3914). A written informed consent was obtained from each patient included in the study and the study protocol conformed to the ethical guidelines of the 1975 Declaration of Helsinki.

\section{Author details \\ ${ }^{1}$ Department of Nutrition, Institute of Basic Medical Sciences, University of Oslo, P.O. Box 1046Blindern, 0317 Oslo, Norway. ${ }^{2}$ Lipid Clinic, Oslo University Hospital, Oslo, Norway. ${ }^{3}$ National Advisory Unit on Familial Hypercholesterolemia, Oslo University Hospital, Oslo, Norway. ${ }^{4}$ The Norwegian Institute of Public Health, Oslo, Norway. ${ }^{5}$ Unit for Cardiac and Cardiovascular Genetics, Department of Medical Genetics, Oslo University Hospital, Oslo, Norway. ${ }^{6}$ Research Institute of Internal Medicine, Oslo University Hospital, Oslo, Norway. ${ }^{7}$ K.G. Jebsen Inflammatory Research Center, University of Oslo, Oslo, Norway. ${ }^{8}$ Section of Clinical Immunology and Infectious Diseases, Oslo University Hospital, Oslo, Norway. ${ }^{9}$ Department of Haematology, Oslo University Hospital, Oslo, Norway.}

Received: 27 March 2017 Accepted: 4 June 2017

Published online: 12 June 2017

\section{References}

1. Sacks FM, Stanesa M, Hegele RA. Severe hypertriglyceridemia with pancreatitis: thirteen years' treatment with lomitapide. JAMA. 2014;174:443-7. 
2. Salmon F, Grosios K, Petry H. Safety profile of recombinant adeno-associated viral vectors: focus on alipogene tiparvovec (Glybera(R)). Exp Rev Clin Pharacol. 2014;7:53-65.

3. Sahebkar A, Chew GT, Watts GF. Recent advances in pharmacotherapy for hypertriglyceridemia. Prog Lipid Res. 2014;56:47-66.

4. Naess O, Sogaard AJ, Arnesen E, Beckstrom AC, Bjertness E, Engeland A, et al. Cohort profile: cohort of Norway (CONOR). Int J Epidemiol. 2008;37:481-5.

5. Tverdal A, Foss OP, Leren P, Holme I, Lund-Larsen PG, Bjartveit K. Serum triglycerides as an independent risk factor for death from coronary heart disease in middle-aged Norwegian men. Am J Epidemiol. 1989;129:458-65.

6. Bjartveit K, Foss OP, Gjervig T, Lund-Larsen PG. The cardiovascular disease study in Norwegian counties. Background and organization. Acta Med Scand. 1979;634:1-70

7. Tverdal A, Hjellvik AV, Selmer R. Coffee intake and oral-oesophageal cancer: follow-up of 389,624 Norwegian men and women 40-45 years. Br J Cancer. 2011;105:157-61.

8. Svilaas A, Strom EC, Svilaas T, Borgejordet A, Thoresen M, Ose L. Reproducibility and validity of a short food questionnaire for the assessment of dietary habits. Nutrition, metabolism, and cardiovascular diseases. NMCD. 2002;12:60-70.

9. Livak KJ, Schmittgen TD. Analysis of relative gene expression data using real-time quantitative PCR and the 2(-Delta Delta $C(T))$ method. Methods. 2001;25:402-8

10. Pedersen $\mathrm{SB}$, Langsted A, Nordestgaard BG. Nonfasting mild-to-moderate hypertriglyceridemia and risk of acute pancreatitis. JAMA Int Med. 2016; 176:1834-42.

11. Berge KE, Retterstol K, Romeo S, Pirazzi C, Leren TP. Type 1 hyperlipoproteinemia due to a novel deletion of exons 3 and 4 in the GPIHBP1 gene. Atherosclerosis. 2014;234:30-3.

12. Konieczna J, Sanchez J, van Schothorst EM, Torrens JM, Bunschoten A, Palou $\mathrm{M}$, et al. Identification of early transcriptome-based biomarkers related to lipid metabolism in peripheral blood mononuclear cells of rats nutritionally programmed for improved metabolic health. Genes Nutr. 2014;9:366.

13. Jung UJ, Seo YR, Ryu R, Choi MS. Differences in metabolic biomarkers in the blood and gene expression profiles of peripheral blood mononuclear cells among normal weight, mildly obese and moderately obese subjects. Br J Nutr. 2016;116:1022-32.

14. Christian JB, Bourgeois N, Snipes R, Lowe KA. Prevalence of severe (500 to $2,000 \mathrm{mg} / \mathrm{dl}$ ) hypertriglyceridemia in United States adults. Am J Cardiol. 2011;107:891-7.

15. Esteghamati A, Hafezi-Nejad N, Sheikhbahaei S, Heidari B, Zandieh A, Ebadi $\mathrm{M}$, et al. Risk of coronary heart disease associated with metabolic syndrome and its individual components in Iranian subjects: a matched cohort study. $J$ Clin Lipid. 2014:8:279-86

16. $\mathrm{Ng} \mathrm{N}$, Johnson O, Lindahl B, Norberg M. A reversal of decreasing trends in population cholesterol levels in Vasterbotten County. Sweden Glob Health Action. 2012;5

17. Kushner PA, Cobble ME. Hypertriglyceridemia: the importance of identifying patients at risk. Postgrad Med. 2016;128:848-58.

18. Stefanutti C, Julius U. Treatment of primary hypertriglyceridemia states-general approach and the role of extracorporeal methods. Atherosclerosis. 2015;18:85-94.

19. Oh RC, Lanier JB. Management of hypertriglyceridemia. Am Fam Phys. 2007; 75:1365-71.

20. Scherer J, Singh VP, Pitchumoni CS, Yadav D. Issues in hypertriglyceridemic pancreatitis: an update. J Clin Gastroenterol. 2014;48:195-203.

21. Joshi M, Eagan J, Desai NK, Newton SA, Towne MC, Marinakis NS, et al. A compound heterozygous mutation in GPD1 causes hepatomegaly, steatohepatitis, and hypertriglyceridemia. Eur J Hum Genet. 2014;22:1229-32.

22. Ferreira MR, Camberos Mdel C, Selenscig D, Martucci LC, Chicco A, Lombardo YB, et al. Changes in hepatic lipogenic and oxidative enzymes and glucose homeostasis induced by an acetyl-L-carnitine and nicotinamide treatment in dyslipidaemic insulin-resistant rats. Clin Exp Pharm Physiol. 2013:40:205-11.

\section{Submit your next manuscript to BioMed Central and we will help you at every step:}

- We accept pre-submission inquiries

- Our selector tool helps you to find the most relevant journal

- We provide round the clock customer support

- Convenient online submission

- Thorough peer review

- Inclusion in PubMed and all major indexing services

- Maximum visibility for your research

Submit your manuscript at www.biomedcentral.com/submit
Biomed Central 